\title{
Metric Characterizations of $\alpha$-Well-Posedness for a System of Mixed Quasivariational-Like Inequalities in Banach Spaces
}

\author{
L. C. Ceng ${ }^{1,2}$ and Y. C. Lin $^{3}$ \\ ${ }^{1}$ Department of Mathematics, Shanghai Normal University, Shanghai 200234, China \\ ${ }^{2}$ Scientific Computing Key Laboratory of Shanghai Universities, Shanghai 200234, China \\ ${ }^{3}$ Department of Occupational Safety and Health, College of Public Health, China Medical University, \\ Taichung 404, Taiwan \\ Correspondence should be addressed to Y. C. Lin, yclin@mail.cmu.edu.tw
}

Received 2 October 2011; Accepted 9 October 2011

Academic Editor: Yonghong Yao

Copyright (C) 2012 L. C. Ceng and Y. C. Lin. This is an open access article distributed under the Creative Commons Attribution License, which permits unrestricted use, distribution, and reproduction in any medium, provided the original work is properly cited.

\begin{abstract}
The purpose of this paper is to investigate the problems of the well-posedness for a system of mixed quasivariational-like inequalities in Banach spaces. First, we generalize the concept of $\alpha$-well-posedness to the system of mixed quasivariational-like inequalities, which includes symmetric quasi-equilibrium problems as a special case. Second, we establish some metric characterizations of $\alpha$-well-posedness for the system of mixed quasivariational-like inequalities. Under some suitable conditions, we prove that the $\alpha$-well-posedness is equivalent to the existence and uniqueness of solution for the system of mixed quasivariational-like inequalities. The corresponding concept of $\alpha$-well-posedness in the generalized sense is also considered for the system of mixed quasivariational-like inequalities having more than one solution. The results presented in this paper generalize and improve some known results in the literature.
\end{abstract}

\section{Introduction}

The classical notion of well-posedness for a minimization problem (MP) is due to Tykhonov [1], which has already been known as the Tykhonov well-posedness. The so-called Tykhonov well-posedness means the existence and uniqueness of solution, and the convergence of every minimizing sequence toward the unique solution. Taking into account that in many practical situations the solution may not be unique for a minimization problem, ones naturally introduced the concept of well-posedness in the generalized sense, which means the existence of minimizers and the convergence of some subsequence of every minimizing sequence toward a minimizer. Obviously, the concept of well-posedness is inspired by numerical methods 
producing optimizing sequences for optimization problems. In the following years, the wellposedness has received much attention because it plays a crucial role in the stability theory for optimization problems. A large number of results about well-posedness have appeared in the literature; see, for example, [2-10], where the work in $[2,3,5,7,10]$ is for the class of scalar optimization problems, and the work in $[4,6,8,11]$ is for the class of vector optimization problems.

On the other hand, the concept of well-posedness has been generalized to other related problems, such as variational inequalities [9, 12-22], Nash equilibrium problems [16, 23-25], inclusion problems $[12,14,26,27]$, and fixed-point problems [12, 14, 26, 28, 29]. An initial notion of well-posedness for variational inequalities is due to Lucchetti and Patrone [20]. They introduced the notion of well-posedness for variational inequalities and proved some related results by means of Ekeland's variational principle. Since then, many authors have been devoted to generating the concept of well-posedness from the minimization problem to various variational inequalities. Lignola and Morgan [19] introduced the parametric well-posedness for a family of variational inequalities. Lignola [15] further introduced two concepts of well-posedness and $L$-well-posedness for quasivariational-like inequalities and derived some metric characterizations of well-posedness. At the same time, Del Prete et al. [18] introduced the concept of $\alpha$-well-posedness for a class of variational inequalities. Recently, Fang et al. [14] generalized the concept of well-posedness to a class of mixed variational inequalities in Hilbert spaces. They obtained some metric characterizations of its well-posedness and established the links with the well-posedness of inclusion problems and fixed-point problems. Furthermore, Ceng and Yao [12] generalized the results of Fang et al. [14] to a class of generalized mixed variational inequalities in Hilbert spaces. Ceng et al. [13] investigated the well-posedness for a class of mixed quasivariational-like inequalities in Banach spaces. For the well-posedness of variational inequalities with functional constraints, we refer to Huang and Yang [9] and Huang et al. [17]. In 2006, Lignola and Morgan [23] presented the notion of $\alpha$-well-posedness for the Nash equilibrium problem and gave some metric characterizations of this type well-posedness. Petruşel et al. [29] and Llorens-Fuster et al. [28] discussed the well-posedness of fixed-point problems for multivalued mappings in metric spaces.

It is obvious that the equilibrium problem plays a very important role in the establishment of a general mathematical model for a wide range of practical problems, which include as special cases optimization problems, Nash equilibria problems, fixed-point problems, variational inequality problems, and complementarity problems (see, e.g, [30,31]), and has been studied extensively and intensively. It is well known that each equilibrium problem can equivalently be transformed into a minimizing problem by using gap function, and some numerical methods have been extended to solve the equilibrium problem (see, e.g., [32]). This fact motivates the researchers to study the well-posedness for equilibrium problems. Recently, Fang et al. [33] introduced the concepts of parametric well-posedness for equilibrium problems and derived some metric characterizations for these types of well-posedness. For the well-posedness of equilibrium problems with functional constraints, we refer the readers to [34]. In 2009, Long and Huang [35] generalized the concept of $\alpha$-wellposedness to symmetric quasiequilibrium problems in Banach spaces, which includes equilibrium problems, Nash equilibrium problems, quasivariational inequalities, variational inequalities, and fixed-point problems as special cases. Under some suitable conditions, they established some metric characterizations of $\alpha$-well-posedness for symmetric quasiequilibrium problems. Moreover, they gave some examples to illustrate their results. Their results represent the generalization and improvement of some previously known results in 
the literature, for instance, $[12-15,23,33]$. It is worth pointing out that up to the publication of [35] there are no results concerned with the problems of the well-posedness for symmetric quasiequilibrium problems in Banach spaces.

In this paper, we consider and study the problems of the well-posedness for a system of mixed quasivariational-like inequalities in Banach spaces. First, we generalize the concept of $\alpha$-well-posedness to the system of mixed quasivariational-like inequalities, which include symmetric quasiequilibrium problems as a special case. Second, some metric characterizations of $\alpha$-well-posedness for the system of mixed quasivariational-like inequalities are given under very mild conditions. Furthermore, it is also proven that under quite appropriate conditions, the $\alpha$-well-posedness is equivalent to the existence and uniqueness of solution for the system of mixed quasivariational-like inequalities. At the same time, the corresponding concept of $\alpha$-well-posedness in the generalized sense is also considered for the system of mixed quasivariational-like inequalities having more than one solution. In addition, we give some examples to illustrate our results. The results presented in this paper generalize and improve Long and Huang's results in [35].

\section{Preliminaries}

Throughout this paper, unless specified otherwise, let $X$ and $Y$ be two real Banach spaces, let their dual spaces be denoted by $X^{*}$ and $Y^{*}$, respectively, and let the duality pairing between $X$ and $X^{*}$ and the one between $Y$ and $Y^{*}$ be denoted by the same $\langle\cdot, \cdot\rangle$. We write $x_{n} \rightarrow x$ to indicate that the sequence $\left\{x_{n}\right\}$ converges weakly to $x$. However, $x_{n} \rightarrow x$ implies that $\left\{x_{n}\right\}$ converges strongly to $x$. Let $C \subset X$ and $D \subset Y$ be two nonempty closed and convex subsets. Let $S: C \times D \rightarrow 2^{C}$ and $T: C \times D \rightarrow 2^{D}$ be two set-valued mappings, let $A: C \times D \rightarrow X^{*}$, $B: C \times D \rightarrow Y^{*}, \hat{\eta}: C \times C \rightarrow X$ and $\bar{\eta}: D \times D \rightarrow Y$ be four single-valued mappings, and let $f, g: C \times D \rightarrow \mathbf{R}$ be two real-valued functions. Suppose that $\alpha$ is a nonnegative real number and $N=\{1,2, \ldots\}$.

In this paper, we consider the system of mixed quasivariational-like inequalities (SMQVLIs), which is to find a point $\left(x_{0}, y_{0}\right) \in C \times D$ such that

$$
\begin{array}{cl}
x_{0} \in S\left(x_{0}, y_{0}\right), & \left\langle A\left(x_{0}, y_{0}\right), \widehat{\eta}\left(x_{0}, z\right)\right\rangle+f\left(x_{0}, y_{0}\right)-f\left(z, y_{0}\right) \leq 0, \quad \forall z \in S\left(x_{0}, y_{0}\right), \\
y_{0} \in T\left(x_{0}, y_{0}\right), & \left\langle B\left(x_{0}, y_{0}\right), \bar{\eta}\left(y_{0}, w\right)\right\rangle+g\left(x_{0}, y_{0}\right)-g\left(x_{0}, w\right) \leq 0, \quad \forall w \in T\left(x_{0}, y_{0}\right) .
\end{array}
$$

Remark 2.1. Whenever $A=0, B=0, \hat{\eta}=0$, and $\bar{\eta}=0$, the Problem (2.1) reduces to the following symmetric quasiequilibrium problem (in short, SQEP) of finding a point $\left(x_{0}, y_{0}\right) \in$ $C \times D$ such that

$$
\begin{array}{ll}
x_{0} \in S\left(x_{0}, y_{0}\right), & f\left(x_{0}, y_{0}\right) \leq f\left(z, y_{0}\right), \quad \forall z \in S\left(x_{0}, y_{0}\right), \\
y_{0} \in T\left(x_{0}, y_{0}\right), & g\left(x_{0}, y_{0}\right) \leq g\left(x_{0}, w\right), \quad \forall w \in T\left(x_{0}, y_{0}\right) .
\end{array}
$$

This problem was first considered by Noor and Oettli [21], which includes equilibrium problems [30], Nash equilibrium problems [36], quasivariational inequalities [37], variational inequalities [38], and fixed-point problems [28, 29] as special cases. It is worth mentioning that Noor and Oettli [21] only established the existence of solutions for SQEP 
(2.2). Subsequently, Long and Huang [35] investigated the $\alpha$-well-posedness for SQEP (2.2) in Banach spaces.

Denote by $\Gamma$ the solution set of SMQVLI (2.1). In what follows, we introduce the notions of $\alpha$-approximating sequence and $\alpha$-well-posedness for SMQVLI (2.1).

Definition 2.2. A sequence $\left\{\left(x_{n}, y_{n}\right)\right\} \subset C \times D$ is called an $\alpha$-approximating sequence for SMQVLI (2.1) if there exists a sequence $\varepsilon_{n}>0$ with $\varepsilon_{n} \rightarrow 0$ such that

$$
\begin{gathered}
d\left(x_{n}, S\left(x_{n}, y_{n}\right)\right) \leq \varepsilon_{n}, \text { that is, } x_{n} \in B\left(S\left(x_{n}, y_{n}\right), \varepsilon_{n}\right), \quad \forall n \in N, \\
d\left(y_{n}, T\left(x_{n}, y_{n}\right)\right) \leq \varepsilon_{n}, \text { that is, } y_{n} \in B\left(T\left(x_{n}, y_{n}\right), \varepsilon_{n}\right), \quad \forall n \in N, \\
\left\langle A\left(x_{n}, y_{n}\right), \hat{\eta}\left(x_{n}, z\right)\right\rangle+f\left(x_{n}, y_{n}\right)-f\left(z, y_{n}\right) \leq \varepsilon_{n}+\frac{\alpha}{2}\left\|x_{n}-z\right\|^{2}, \quad \forall z \in S\left(x_{n}, y_{n}\right), \quad \forall n \in N, \\
\left\langle B\left(x_{n}, y_{n}\right), \bar{\eta}\left(y_{n}, w\right)\right\rangle+g\left(x_{n}, y_{n}\right)-g\left(x_{n}, w\right) \leq \varepsilon_{n}+\frac{\alpha}{2}\left\|y_{n}-w\right\|^{2}, \quad \forall w \in T\left(x_{n}, y_{n}\right), \quad \forall n \in N,
\end{gathered}
$$

where $B(S(x, y), \varepsilon)$ denotes the ball of radius $\varepsilon$ around $S(x, y)$, that is, the set $\{m \in X$ : $\left.d(S(x, y), m)=\inf _{b \in S(x, y)}\|m-b\| \leq \varepsilon_{n}\right\}$. Whenever $\alpha=0$, one says that the sequence $\left\{\left(x_{n}, y_{n}\right)\right\}$ is an approximating sequence for SMQVLI (2.1).

We remark that if $A=0, B=0, \hat{\eta}=0$, and $\bar{\eta}=0$, the notions of $\alpha$-approximating sequence and approximating sequence for SMQVLI (2.1) reduce to the ones of $\alpha$ approximating sequence and approximating sequence for SQEP (2.2) in [35, Definition 2.1], respectively.

Definition 2.3. SMQVLI (2.1) is said to be $\alpha$-well-posed if it has a unique solution $\left(x_{0}, y_{0}\right)$ and every $\alpha$-approximating sequence $\left\{\left(x_{n}, y_{n}\right)\right\}$ converges strongly to $\left(x_{0}, y_{0}\right)$. Whenever $\alpha=0$, we say that SMQVLI (2.1) is well-posed.

We remark that if $A=0, B=0, \hat{\eta}=0$, and $\bar{\eta}=0$, the notions of $\alpha$-well-posedness and well-posedness for SMQVLI (2.1) reduce to the ones of $\alpha$-well-posedness and well-posedness for SQEP (2.2) in [35, Definition 2.2], respectively.

Definition 2.4. SMQVLI (2.1) is said to be $\alpha$-well-posed in the generalized sense if the solution set $\Gamma$ of SMQVLI (2.1) is nonempty and every $\alpha$-approximating sequence $\left\{\left(x_{n}, y_{n}\right)\right\}$ has a subsequence which converges strongly to some element of $\Gamma$. Whenever $\alpha=0$, one says that SMQVLI (2.1) is well-posed in the generalized sense.

We remark that if $A=0, B=0, \hat{\eta}=0$, and $\bar{\eta}=0$, the notions of $\alpha$-well-posedness in the generalized sense and well-posedness in the generalized sense for SMQVLI (2.1) reduce to the ones of $\alpha$-well-posedness in the generalized sense and well-posedness in the generalized sense for SQEP (2.2) in [35, Definition 2.3], respectively.

In order to investigate the $\alpha$-well-posedness for SMQVLI (2.1), we need the following definitions. 
Definition 2.5 (see [39]). The Painleve-Kuratowski limits of a sequence $\left\{H_{n}\right\} \subset X$ are defined by

$$
\liminf _{n} H_{n}=\left\{y \in X: \exists y_{n} \in H_{n}, n \in N \text {, with } \lim _{n} y_{n}=y\right\}
$$

$\lim \sup _{n} H_{n}=\left\{y \in X: \exists n_{k} \uparrow+\infty, n_{k} \in N, \exists y_{n_{k}} \in H_{n_{k}}, k \in N\right.$, with $\left.\lim _{k} y_{n_{k}}=y\right\}$.

Definition 2.6 (see [39]). A set-valued mapping $F$ from a topological space $(W, \tau)$ to a topological space $(Z, \sigma)$ is called

(i) $(\tau, \sigma)$-closed if for every $\mathrm{x} \in \mathrm{K}$, for every sequence $\left\{x_{n}\right\} \tau$-converging to $x$, and for every sequence $\left\{y_{n}\right\} \sigma$-converging to a point $y$, such that $y_{n} \in F\left(x_{n}\right)$, one has $y \in F(x)$, that is,

$$
F(x) \supset \lim _{n} \sup F\left(x_{n}\right),
$$

(ii) $(\tau, \sigma)$-lower semicontinuous if for every $\mathrm{x} \in \mathrm{K}$, for every sequence $\left\{x_{n}\right\} \tau$ converging to $x$, and for every $y \in F(x)$, there exists a sequence $\left\{y_{n}\right\} \sigma$-converging to $y$, such that $y_{n} \in F\left(x_{n}\right)$ for $n$ sufficiently large, that is,

$$
F(x) \subset \lim _{n} \inf F\left(x_{n}\right),
$$

(iii) $(\tau, \sigma)$-subcontinuous on $K$, if for every sequence $\left\{x_{n}\right\} \tau$-converging in $K$, every sequence $\left\{y_{n}\right\}$, such that $y_{n} \in F\left(x_{n}\right)$, has a $\sigma$-convergent subsequence.

Definition 2.7 (see [39]). Let $V$ be a nonempty subset of $X$. The measure of noncompactness $\mu$ of the set $V$ is defined by

$$
\mu(V)=\inf \left\{\varepsilon>0: V \subset \bigcup_{i=1}^{n} V_{i}, \operatorname{diam} V_{i}<\varepsilon, i=1,2, \ldots, n\right\},
$$

where diam means the diameter of a set.

Definition 2.8 (see [39]). Let $(X, d)$ be a metric space and let $U, V$ be nonempty subsets of $X$. The Hausdorff metric $\mathscr{\ell}(\cdot, \cdot)$ between $U$ and $V$ is defined by

$$
\mathscr{H}(U, V)=\max \{e(U, V), e(V, U)\}
$$

where $e(U, V)=\sup _{u \in U} d(u, V)$ with $d(u, V)=\inf _{v \in V}\|u-v\|$. Let $\left\{U_{n}\right\}$ be a sequence of nonempty subsets of $X$. One says that $U_{n}$ converges to $U$ in the sense of Hausdorff metric if 
$\mathscr{\ell}\left(U_{n}, U\right) \rightarrow 0$. It is easy to see that $e\left(U_{n}, U\right) \rightarrow 0$ if and only if $d\left(u_{n}, U\right) \rightarrow 0$ for all section $u_{n} \in U_{n}$. For more details on this topic, the readers refered one to [39].

Now, we prove the following lemma.

Lemma 2.9. Suppose that set-valued mappings $S$ and $T$ are nonempty convex-valued, the function $f(\cdot, y)$ is convex on $C$ for any $y \in D$, and the function $g(x, \cdot)$ is convex on $D$ for any $x \in C$. Then $\left(x_{0}, y_{0}\right) \in \Gamma$ if and only if the following two conditions hold:

$$
\begin{array}{r}
x_{0} \in S\left(x_{0}, y_{0}\right), \quad\left\langle A\left(x_{0}, y_{0}\right), \widehat{\eta}\left(x_{0}, z\right)\right\rangle+f\left(x_{0}, y_{0}\right)-f\left(z, y_{0}\right) \leq \frac{\alpha}{2}\left\|x_{0}-z\right\|^{2}, \\
\forall z \in S\left(x_{0}, y_{0}\right), \\
y_{0} \in T\left(x_{0}, y_{0}\right), \quad\left\langle B\left(x_{0}, y_{0}\right), \bar{\eta}\left(y_{0}, w\right)\right\rangle+g\left(x_{0}, y_{0}\right)-g\left(x_{0}, w\right) \leq \frac{\alpha}{2}\left\|y_{0}-w\right\|^{2}, \\
\forall w \in T\left(x_{0}, y_{0}\right),
\end{array}
$$

where both $\hat{\eta}: C \times C \rightarrow X$ and $\bar{\eta}: D \times D \rightarrow Y$ are affine in the second variable such that $\hat{\eta}(x, x)=0$ and $\bar{\eta}(y, y)=0$ for all $(x, y) \in C \times D$.

Proof. The necessity is obvious. For the sufficiency, suppose that (2.9) holds. Now let us show that $\left(x_{0}, y_{0}\right) \in \Gamma$. Indeed, let $z \in S\left(x_{0}, y_{0}\right)$ and for any $t \in[0,1], z_{t}=t z+(1-t) x_{0}$. Since $S\left(x_{0}, y_{0}\right)$ is convex, $z_{t} \in S\left(x_{0}, y_{0}\right)$ and so

$$
\left\langle A\left(x_{0}, y_{0}\right), \widehat{\eta}\left(x_{0}, z_{t}\right)\right\rangle+f\left(x_{0}, y_{0}\right)-f\left(z_{t}, y_{0}\right) \leq \frac{\alpha}{2}\left\|x_{0}-z_{t}\right\|^{2}, \quad \forall t \in(0,1]
$$

Also, since $f(\cdot, y)$ is convex for any $y \in D$ and $\hat{\eta}: C \times C \rightarrow X$ is affine in the second variable with $\widehat{\eta}(x, x)=0, \forall x \in X$, we have

$$
\begin{aligned}
& t\left\{\left\langle A\left(x_{0}, y_{0}\right), \widehat{\eta}\left(x_{0}, z\right)\right\rangle+f\left(x_{0}, y_{0}\right)-f\left(z, y_{0}\right)\right\} \\
&=\left\langle A\left(x_{0}, y_{0}\right), t \hat{\eta}\left(x_{0}, z\right)+(1-t) \widehat{\eta}\left(x_{0}, x_{0}\right)\right\rangle \\
&+f\left(x_{0}, y_{0}\right)-\left(t f\left(z, y_{0}\right)+(1-t) f\left(x_{0}, y_{0}\right)\right) \\
& \leq\left\langle A\left(x_{0}, y_{0}\right), \widehat{\eta}\left(x_{0}, z_{t}\right)\right\rangle+f\left(x_{0}, y_{0}\right)-f\left(z_{t}, y_{0}\right) \\
& \leq \frac{\alpha}{2}\left\|x_{0}-z_{t}\right\|^{2} \\
&= \frac{\alpha}{2} t^{2}\left\|x_{0}-z\right\|^{2}, \quad \forall t \in(0,1] .
\end{aligned}
$$

Thus, dividing by $t$ in the above inequality, we have

$$
\left\langle A\left(x_{0}, y_{0}\right), \widehat{\eta}\left(x_{0}, z\right)\right\rangle+f\left(x_{0}, y_{0}\right)-f\left(z, y_{0}\right) \leq \frac{\alpha}{2} t\left\|x_{0}-z\right\|^{2}, \quad \forall t \in(0,1], z \in S\left(x_{0}, y_{0}\right)
$$


By the similar argument,

$$
\left\langle B\left(x_{0}, y_{0}\right), \bar{\eta}\left(y_{0}, w\right)\right\rangle+g\left(x_{0}, y_{0}\right)-g\left(x_{0}, w\right) \leq \frac{\alpha}{2} t\left\|y_{0}-w\right\|^{2}, \quad \forall t \in(0,1], w \in T\left(x_{0}, y_{0}\right) .
$$

The combination of (2.12) and (2.13) implies, for $t$ tending to zero, that $\left(x_{0}, y_{0}\right)$ is a solution of SMQVLI (2.1). This completes the proof.

Corollary 2.10 (i.e., [35, Lemma 2.1]). Suppose that set-valued mappings $S$ and $T$ are nonempty convex-valued, the function $f(\cdot, y)$ is convex on $C$ for any $y \in D$, and the function $g(x, \cdot)$ is convex on $D$ for any $x \in C$. Then $\left(x_{0}, y_{0}\right)$ solves SQEP (2.2) if and only if the following two conditions hold:

$$
\begin{gathered}
x_{0} \in S\left(x_{0}, y_{0}\right), \quad f\left(x_{0}, y_{0}\right) \leq f\left(z, y_{0}\right)+\frac{\alpha}{2}\left\|x_{0}-z\right\|^{2}, \quad \forall z \in S\left(x_{0}, y_{0}\right), \\
y_{0} \in T\left(x_{0}, y_{0}\right), \quad g\left(x_{0}, y_{0}\right) \leq g\left(x_{0}, w\right)+\frac{\alpha}{2}\left\|y_{0}-w\right\|^{2}, \quad \forall w \in T\left(x_{0}, y_{0}\right) .
\end{gathered}
$$

Proof. Put $A=0, B=0, \hat{\eta}=0$, and $\bar{\eta}=0$ in Lemma 2.9. Then, utilizing Lemma 2.9 we get the desired result.

\section{Metric Characterizations of $\alpha$-Well-Posedness for SMQVLI}

In this section, we will investigate some metric characterizations of $\alpha$-well-posedness for SMQVLI (2.1).

For any $\varepsilon>0$, the $\alpha$-approximating solution set of SMQVLI (2.1) is defined by

$$
\begin{aligned}
\Omega_{\alpha}(\varepsilon)=\{ & \left(x_{0}, y_{0}\right) \in C \times D: \\
& x_{0} \in B\left(S\left(x_{0}, y_{0}\right), \varepsilon\right),\left\langle A\left(x_{0}, y_{0}\right), \widehat{\eta}\left(x_{0}, z\right)\right\rangle+f\left(x_{0}, y_{0}\right)-f\left(z, y_{0}\right) \\
& \leq \varepsilon+\frac{\alpha}{2}\left\|x_{0}-z\right\|^{2}, \forall z \in S\left(x_{0}, y_{0}\right), y_{0} \in B\left(T\left(x_{0}, y_{0}\right), \varepsilon\right),\left\langle B\left(x_{0}, y_{0}\right), \bar{\eta}\left(y_{0}, w\right)\right\rangle \\
& \left.+g\left(x_{0}, y_{0}\right)-g\left(x_{0}, w\right) \leq \varepsilon+\frac{\alpha}{2}\left\|y_{0}-w\right\|^{2}, \forall w \in T\left(x_{0}, y_{0}\right)\right\} .
\end{aligned}
$$

Theorem 3.1. SMQVLI (2.1) is $\alpha$-well-posed if and only if the solution set $\Gamma$ of SMQVLI (2.1) is nonempty and

$$
\lim _{\varepsilon \rightarrow 0} \operatorname{diam} \Omega_{\alpha}(\varepsilon)=0
$$

Proof. Suppose that SMQVLI (2.1) is $\alpha$-well-posed. Then, $\Gamma$ is a singleton, and $\Omega_{\alpha}(\varepsilon) \neq \emptyset$ for any $\varepsilon>0$, since $\Gamma \subset \Omega_{\alpha}(\varepsilon)$. Suppose by contraction that

$$
\lim _{\varepsilon \rightarrow 0} \operatorname{diam} \Omega_{\alpha}(\varepsilon)>\beta>0
$$


Then there exists $\varepsilon_{n}>0$ with $\varepsilon_{n} \rightarrow 0$, and $\left(x_{n}, y_{n}\right),\left(\bar{x}_{n}, \bar{y}_{n}\right) \in \Omega_{\alpha}\left(\varepsilon_{n}\right)$ such that

$$
\left\|\left(x_{n}, y_{n}\right)-\left(\bar{x}_{n}, \bar{y}_{n}\right)\right\|>\beta, \quad \forall n \in N
$$

where the norm $\|\cdot\|$ in the product space $X \times Y$ is defined as follows:

$$
\|(u, v)-(\bar{u}, \bar{v})\|=\sqrt{\|u-\bar{u}\|^{2}+\|v-\bar{v}\|^{2}}, \quad \forall(u, v),(\bar{u}, \bar{v}) \in X \times Y .
$$

(It is not difficult to verify that $X \times Y$ is a Banach space in terms of the last norm.)

Since $\left(x_{n}, y_{n}\right),\left(\bar{x}_{n}, \bar{y}_{n}\right) \in \Omega_{\alpha}\left(\varepsilon_{n}\right)$, and SMQVLI (2.1) is $\alpha$-well-posed, the sequences $\left\{\left(x_{n}, y_{n}\right)\right\}$ and $\left\{\left(\bar{x}_{n}, \bar{y}_{n}\right)\right\}$, which are both $\alpha$-approximating sequences for SMQVLI (2.1), converge strongly to the unique solution $\left(x_{0}, y_{0}\right)$, and this leads to a contraction. Therefore, (3.2) holds.

Conversely, let (3.2) hold and let $\left\{\left(x_{n}, y_{n}\right)\right\} \subset C \times D$ be any $\alpha$-approximating sequence for SMQVLI (2.1). Then, there exists a sequence $\varepsilon_{n}>0$ with $\varepsilon_{n} \rightarrow 0$ such that

$$
\begin{array}{r}
d\left(x_{n}, S\left(x_{n}, y_{n}\right)\right) \leq \varepsilon_{n}, \quad\left\langle A\left(x_{n}, y_{n}\right), \widehat{\eta}\left(x_{n}, z\right)\right\rangle+f\left(x_{n}, y_{n}\right)-f\left(z, y_{n}\right) \leq \varepsilon_{n}+\frac{\alpha}{2}\left\|x_{n}-z\right\|^{2}, \\
\forall z \in S\left(x_{n}, y_{n}\right), \\
d\left(y_{n}, T\left(x_{n}, y_{n}\right)\right) \leq \varepsilon_{n}, \quad\left\langle B\left(x_{n}, y_{n}\right), \bar{\eta}\left(y_{n}, w\right)\right\rangle+g\left(x_{n}, y_{n}\right)-g\left(x_{n}, w\right) \leq \varepsilon_{n}+\frac{\alpha}{2}\left\|y_{n}-w\right\|^{2}, \\
\forall w \in T\left(x_{n}, y_{n}\right) .
\end{array}
$$

This implies that $\left\{\left(x_{n}, y_{n}\right)\right\} \subset \Omega_{\alpha}\left(\varepsilon_{n}\right)$, for all $n \in N$. Since the solution set $\Gamma$ of SMQVLI (2.1) is nonempty, we can take two elements in $\Gamma$ arbitrarily, denoted by $\left(x_{0}, y_{0}\right)$ and $\left(\bar{x}_{0}, \bar{y}_{0}\right)$, respectively. Note that $\Gamma \subset \Omega_{\alpha}(\varepsilon)$ for all $\varepsilon>0$. Hence both $\left(x_{0}, y_{0}\right)$ and $\left(\bar{x}_{0}, \bar{y}_{0}\right)$ lie in $\Omega_{\alpha}\left(\varepsilon_{n}\right)$ for all $n \geq 1$. This fact together with (3.2) yields

$$
\left\|\left(x_{n}, y_{n}\right)-\left(x_{0}, y_{0}\right)\right\| \leq \operatorname{diam} \Omega_{\alpha}\left(\varepsilon_{n}\right) \longrightarrow 0, \quad\left\|\left(x_{n}, y_{n}\right)-\left(\bar{x}_{0}, \bar{y}_{0}\right)\right\| \leq \operatorname{diam} \Omega_{\alpha}\left(\varepsilon_{n}\right) \longrightarrow 0
$$

Utilizing (3.7) and the uniqueness of the limit, we conclude that $\left(x_{0}, y_{0}\right)=\left(\bar{x}_{0}, \bar{y}_{0}\right)$. This means that $\Gamma$ is a singleton. Thus, it is known that SMQVLI (2.1) has the unique solution $\left(x_{0}, y_{0}\right)$ and $\left\{\left(x_{n}, y_{n}\right)\right\}$ converges strongly to $\left(x_{0}, y_{0}\right)$. This shows that SMQVLI (2.1) is $\alpha$-well-posed. This completes the proof.

Corollary 3.2 (i.e., [35, Theorem 3.1]). SQEP (2.2) is $\alpha$-well-posed if and only if the solution set $\Gamma$ of SQEP (2.2) is nonempty and

$$
\lim _{\varepsilon \rightarrow 0} \operatorname{diam} M_{\varepsilon}=0
$$


where

$$
\begin{aligned}
M_{\varepsilon}=\{ & \left(x_{0}, y_{0}\right) \in C \times D: x_{0} \in B\left(S\left(x_{0}, y_{0}\right), \varepsilon\right), f\left(x_{0}, y_{0}\right)-f\left(z, y_{0}\right) \\
& \leq \varepsilon+\frac{\alpha}{2}\left\|x_{0}-z\right\|^{2}, \forall z \in S\left(x_{0}, y_{0}\right), y_{0} \in B\left(T\left(x_{0}, y_{0}\right), \varepsilon\right), g\left(x_{0}, y_{0}\right)-g\left(x_{0}, w\right) \leq \varepsilon \\
& \left.+\frac{\alpha}{2}\left\|y_{0}-w\right\|^{2}, \forall w \in T\left(x_{0}, y_{0}\right)\right\} .
\end{aligned}
$$

Proof. Put $A=0, B=0, \hat{\eta}=0$, and $\bar{\eta}=0$ in Theorem 3.1. Then, utilizing Theorem 3.1 we get the desired result.

In the sequel, the following concept will be needed to apply to our main results.

Definition 3.3. Let $C$ be a nonempty, closed convex subset of $X$. A single-valued mapping $\eta: C \times C \rightarrow X$ is said to be Lipschitz continuous if there exists a constant $\lambda>0$ such that

$$
\|\eta(x, y)\| \leq \lambda\|x-y\|, \quad \forall x, y \in C .
$$

We remark that whenever $X=H$ a Hilbert space and $C=K$ a nonempty closed convex subset of $H$, the Lipschitz continuous mapping $\eta: K \times K \rightarrow H$ has been introduced and considered in Ansari and Yao [40]. In their main result for the existence of solutions and convergence of iterative algorithm (i.e., [40, Theorem 3.1]), the Lipschitz continuous mapping $\eta: K \times K \rightarrow H$ satisfies the following conditions:

(a) $\eta(x, y)+\eta(y, x)=0$ for all $x, y \in K$,

(b) $\eta(x, y)=\eta(x, z)+\eta(z, y)$ for all $x, y, z \in K$,

(c) $\eta(\cdot, \cdot)$ is affine in the first variable,

(d) for each fixed $y \in K, x \mapsto \eta(y, x)$ is sequentially continuous from the weak topology to the weak topology $((w, w)$-continuous).

Inspired by the above restrictions imposed on the Lipschitz continuous mapping $\eta$, we give the following theorem.

Theorem 3.4. Assume that the following conditions hold:

(i) set-valued mappings $S$ and $T$ are nonempty convex-valued, $(s, w)$-closed, $(s, s)$-lower semicontinuous and $(s, w)$-subcontinuous on $C \times D$;

(ii) single-valued mappings $A$ and $B$ are $\left(s, w^{*}\right)$-continuous on $C \times D$;

(iii) single-valued mappings $\hat{\eta}$ and $\bar{\eta}$ are Lipschitz continuous with constants $\hat{\lambda}$ and $\bar{\lambda}$ respectively, such that

(a) $\hat{\eta}\left(x_{1}, x_{3}\right)=\widehat{\eta}\left(x_{1}, x_{2}\right)+\widehat{\eta}\left(x_{2}, x_{3}\right)$ for all $x_{1}, x_{2}, x_{3} \in C$ and $\bar{\eta}\left(y_{1}, y_{3}\right)=\bar{\eta}\left(y_{1}, y_{2}\right)+$ $\bar{\eta}\left(y_{2}, y_{3}\right)$ for all $y_{1}, y_{2}, y_{3} \in D$,

(b) $\widehat{\eta}(\cdot, \cdot)$ and $\bar{\eta}(\cdot, \cdot)$ both are affine in the second variable;

(iv) functions $f$ and $g$ are continuous on $C \times D$; 
(v) for any $y \in D$, the function $f(\cdot, y)$ is convex on $C$; for any $x \in C$, the function $g(x, \cdot)$ is convex on $D$.

Then, SMQVLI (2.1) is $\alpha$-well-posed if and only if

$$
\Omega_{\alpha}(\varepsilon) \neq \emptyset, \quad \forall \varepsilon>0, \quad \lim _{\varepsilon \rightarrow 0} \operatorname{diam} \Omega_{\alpha}(\varepsilon)=0 .
$$

Proof. First, utilizing condition (iii) (a), we can readily obtain that

$$
\begin{array}{ccc}
\widehat{\eta}\left(x_{1}, x_{1}\right)=0, & \widehat{\eta}\left(x_{1}, x_{2}\right)=-\widehat{\eta}\left(x_{2}, x_{1}\right), & \forall x_{1}, x_{2} \in C \\
\bar{\eta}\left(y_{1}, y_{1}\right)=0, & \bar{\eta}\left(y_{1}, y_{2}\right)=-\bar{\eta}\left(y_{2}, y_{1}\right), & \forall y_{1}, y_{2} \in D .
\end{array}
$$

The necessity has been proved in Theorem 3.1. For the sufficiency, let condition (3.11) hold. Let $\left\{\left(x_{n}, y_{n}\right)\right\} \subset C \times D$ be any $\alpha$-approximating sequence for SMQVLI (2.1). Now let us show that $\Gamma$ is a singleton and $\left\{\left(x_{n}, y_{n}\right)\right\}$ converges strongly to the unique element of $\Gamma$. As a matter of fact, since $\left\{\left(x_{n}, y_{n}\right)\right\}$ is $\alpha$-approximating sequence for SMQVLI (2.1), there exists a sequence $\varepsilon_{n}>0$ with $\varepsilon_{n} \rightarrow 0$ such that

$$
\begin{array}{r}
d\left(x_{n}, S\left(x_{n}, y_{n}\right)\right) \leq \varepsilon_{n}, \quad\left\langle A\left(x_{n}, y_{n}\right), \hat{\eta}\left(x_{n}, z\right)\right\rangle+f\left(x_{n}, y_{n}\right)-f\left(z, y_{n}\right) \leq \varepsilon_{n}+\frac{\alpha}{2}\left\|x_{n}-z\right\|^{2}, \\
\forall z \in S\left(x_{n}, y_{n}\right), \\
d\left(y_{n}, T\left(x_{n}, y_{n}\right)\right) \leq \varepsilon_{n}, \quad\left\langle B\left(x_{n}, y_{n}\right), \bar{\eta}\left(y_{n}, w\right)\right\rangle+g\left(x_{n}, y_{n}\right)-g\left(x_{n}, w\right) \leq \varepsilon_{n}+\frac{\alpha}{2}\left\|y_{n}-w\right\|^{2}, \\
\forall w \in T\left(x_{n}, y_{n}\right) .
\end{array}
$$

This means $\left\{\left(x_{n}, y_{n}\right)\right\} \subset \Omega_{\alpha}\left(\varepsilon_{n}\right)$, for all $n \in N$. It follows from (3.11) that $\left\{\left(x_{n}, y_{n}\right)\right\}$ is a Cauchy sequence in Banach space $(X \times Y,\|\cdot\|)$ and hence converges strongly to a point $\left(x_{0}, y_{0}\right) \in X \times Y$. By the definition of the norm $\|\cdot\|$ in Banach space $(X \times Y,\|\cdot\|)$, we deduce that

$$
\begin{aligned}
& \left\|x_{n}-x_{0}\right\| \leq \sqrt{\left\|x_{n}-x_{0}\right\|^{2}+\left\|y_{n}-y_{0}\right\|^{2}}=\left\|\left(x_{n}, y_{n}\right)-\left(x_{0}, y_{0}\right)\right\| \longrightarrow 0 \\
& \left\|y_{n}-y_{0}\right\| \leq \sqrt{\left\|x_{n}-x_{0}\right\|^{2}+\left\|y_{n}-y_{0}\right\|^{2}}=\left\|\left(x_{n}, y_{n}\right)-\left(x_{0}, y_{0}\right)\right\| \longrightarrow 0
\end{aligned}
$$

On account of the closedness of $C$ and $D$ we conclude from $\left\{x_{n}\right\} \subset C$ and $\left\{y_{n}\right\} \subset D$ that $x_{n} \rightarrow x_{0} \in C$ and $y_{n} \rightarrow y_{0} \in D$. In order to show $\left(x_{0}, y_{0}\right) \in \Gamma$, we start to prove that

$$
d\left(x_{0}, S\left(x_{0}, y_{0}\right)\right) \leq \liminf _{n} d\left(x_{n}, S\left(x_{n}, y_{n}\right)\right)=\lim _{n} \varepsilon_{n}=0
$$

Indeed, suppose that the left inequality does not hold. Then there exists a positive number $\gamma$ such that

$$
\liminf _{n} d\left(x_{n}, S\left(x_{n}, y_{n}\right)\right)<r<d\left(x_{0}, S\left(x_{0}, y_{0}\right)\right)
$$


or equivalently, there exist an increasing sequence $\left\{n_{k}\right\}$ and a sequence $\left\{z_{k}\right\}, z_{k} \in S\left(x_{n_{k}}, y_{n_{k}}\right)$, fo all $k \in N$ such that

$$
\left\|x_{n_{k}}-z_{k}\right\|<\gamma, \quad \forall k \in N
$$

Since the set-valued mapping $S$ is $(s, w)$-closed and $(s, w)$-subcontinuous, the sequence $\left\{z_{k}\right\}$ has a subsequence, denoted still by $\left\{z_{k}\right\}$, converging weakly to a point $z_{0} \in S\left(x_{0}, y_{0}\right)$. From the weak lower semicontinuity of the norm, it follows that

$$
r<d\left(x_{0}, S\left(x_{0}, y_{0}\right)\right) \leq\left\|x_{0}-z_{0}\right\| \leq \liminf _{k}\left\|x_{n_{k}}-z_{k}\right\|<\gamma,
$$

which leads to a contradiction. Thus we must have $d\left(x_{0}, S\left(x_{0}, y_{0}\right)\right)=0$ and hence $x_{0} \in$ $S\left(x_{0}, y_{0}\right)$. Similarly, we can prove $y_{0} \in T\left(x_{0}, y_{0}\right)$.

To complete the proof, we take a point $z \in S\left(x_{0}, y_{0}\right)$ arbitrarily. Since $S$ is $(s, s)$ lower semicontinuous, there exists a sequence $\left\{z_{n}\right\}$ converging strongly to $z$, such that $z_{n} \in S\left(x_{n}, y_{n}\right)$ for $n$ sufficiently large. Furthermore, utilizing condition (iii) (a) and the Lipschitz continuity of $\hat{\eta}$ we deduce that

$$
\begin{aligned}
\left\|\widehat{\eta}\left(x_{n}, z_{n}\right)-\hat{\eta}\left(x_{0}, z\right)\right\| & =\left\|\widehat{\eta}\left(x_{n}, z_{n}\right)-\widehat{\eta}\left(x_{0}, z_{n}\right)+\widehat{\eta}\left(x_{0}, z_{n}\right)-\widehat{\eta}\left(x_{0}, z\right)\right\| \\
& \leq\left\|\hat{\eta}\left(x_{n}, z_{n}\right)-\widehat{\eta}\left(x_{0}, z_{n}\right)\right\|+\left\|\widehat{\eta}\left(x_{0}, z_{n}\right)-\widehat{\eta}\left(x_{0}, z\right)\right\| \\
& =\left\|\hat{\eta}\left(x_{n}, z_{n}\right)+\widehat{\eta}\left(z_{n}, x_{0}\right)\right\|+\left\|\widehat{\eta}\left(x_{0}, z_{n}\right)+\widehat{\eta}\left(z, x_{0}\right)\right\| \\
& =\left\|\hat{\eta}\left(x_{n}, x_{0}\right)\right\|+\left\|\widehat{\eta}\left(z, z_{n}\right)\right\| \\
& \leq \hat{\imath}\left(\left\|x_{n}-x_{0}\right\|+\left\|z_{n}-z\right\|\right) \longrightarrow 0 \quad \text { as } n \longrightarrow \infty .
\end{aligned}
$$

Since $A$ is $\left(s, w^{*}\right)$-continuous, it is known that $A\left(x_{n}, y_{n}\right)$ converges weakly to $A\left(x_{0}, y_{0}\right)$, that is, for each $x \in X$, the real sequence $\left\{\left\langle A\left(x_{n}, y_{n}\right), x\right\rangle\right\}$ converges to the real number $\left\langle A\left(x_{0}, y_{0}\right), x\right\rangle$. This implies that $\left\{\left\langle A\left(x_{n}, y_{n}\right), x\right\rangle\right\}$ is a bounded sequence of real numbers for each $x \in X$. Thus $\left\{A\left(x_{n}, y_{n}\right)\right\}$ is bounded in the norm topology according to the uniform boundedness principle [41], that is, $\sup _{n \geq 1}\left\|A\left(x_{n}, y_{n}\right)\right\|<\infty$.

Now observe that

$$
\begin{aligned}
&\left|\left\langle A\left(x_{n}, y_{n}\right), \widehat{\eta}\left(x_{n}, z_{n}\right)\right\rangle-\left\langle A\left(x_{0}, y_{0}\right), \widehat{\eta}\left(x_{0}, z\right)\right\rangle\right| \\
&=\mid\left\langle A\left(x_{n}, y_{n}\right), \widehat{\eta}\left(x_{n}, z_{n}\right)\right\rangle-\left\langle A\left(x_{n}, y_{n}\right), \widehat{\eta}\left(x_{0}, z\right)\right\rangle \\
& \quad+\left\langle A\left(x_{n}, y_{n}\right), \widehat{\eta}\left(x_{0}, z\right)\right\rangle-\left\langle A\left(x_{0}, y_{0}\right), \widehat{\eta}\left(x_{0}, z\right)\right\rangle \mid \\
& \leq\left|\left\langle A\left(x_{n}, y_{n}\right), \widehat{\eta}\left(x_{n}, z_{n}\right)-\widehat{\eta}\left(x_{0}, z\right)\right\rangle\right|+\left|\left\langle A\left(x_{n}, y_{n}\right)-A\left(x_{0}, y_{0}\right), \widehat{\eta}\left(x_{0}, z\right)\right\rangle\right| \\
& \leq\left\|A\left(x_{n}, y_{n}\right)\right\|\left\|\hat{\eta}\left(x_{n}, z_{n}\right)-\widehat{\eta}\left(x_{0}, z\right)\right\| \\
&+\left|\left\langle A\left(x_{n}, y_{n}\right)-A\left(x_{0}, y_{0}\right), \widehat{\eta}\left(x_{0}, z\right)\right\rangle\right| \longrightarrow 0 \text { as } n \longrightarrow \infty .
\end{aligned}
$$


Consequently, it follows from condition (iv) that

$$
\begin{aligned}
& \left\langle A\left(x_{0}, y_{0}\right), \widehat{\eta}\left(x_{0}, z\right)\right\rangle+f\left(x_{0}, y_{0}\right)-f\left(z, y_{0}\right) \\
& \quad=\lim _{n}\left\{\left\langle A\left(x_{n}, y_{n}\right), \widehat{\eta}\left(x_{n}, z_{n}\right)\right\rangle+f\left(x_{n}, y_{n}\right)-f\left(z_{n}, y_{n}\right)\right\} \\
& \quad \leq \lim _{n}\left(\varepsilon_{n}+\frac{\alpha}{2}\left\|x_{n}-z_{n}\right\|^{2}\right) \\
& \quad=\frac{\alpha}{2}\left\|x_{0}-z\right\|^{2}, \quad \text { for all } z \in S\left(x_{0}, y_{0}\right) .
\end{aligned}
$$

Analogously, we have

$$
\left\langle B\left(x_{0}, y_{0}\right), \bar{\eta}\left(y_{0}, w\right)\right\rangle+g\left(x_{0}, y_{0}\right)-g\left(x_{0}, w\right) \leq \frac{\alpha}{2}\left\|y_{0}-w\right\|^{2}, \quad \forall w \in T\left(x_{0}, y_{0}\right)
$$

It follows from Lemma 2.9 that $\left(x_{0}, y_{0}\right) \in \Gamma$. Therefore, SMQVLI (2.1) is $\alpha$-well-posed. This completes the proof.

Corollary 3.5 (i.e., [35, Theorem 3.2]). Assume that the following conditions hold:

(i) set-valued mappings $S$ and $T$ are nonempty convex-valued, $(s, w)$-closed, $(s, s)$-lower semicontinuous, and $(s, w)$-subcontinuous on $C \times D$;

(ii) functions $f$ and $g$ are continuous on $C \times D$;

(iii) for any $y \in D$, the function $f(\cdot, y)$ is convex on $C$; for any $x \in C$, the function $g(x, \cdot)$ is convex on $D$.

Then, SQEP (2.2) is $\alpha$-well-posed if and only if

$$
M_{\varepsilon} \neq \emptyset, \quad \forall \varepsilon>0, \quad \lim _{\varepsilon \rightarrow 0} \operatorname{diam} M_{\varepsilon}=0 .
$$

To illustrate Theorem 3.4, we give the following two examples.

Example 3.6. Let $X=Y=\mathbf{R}$ and $C=D=\mathbf{R}^{+}(=[0,+\infty))$. Let $S(x, y)=[0, x], T(x, y)=[0, y]$, $A(x, y)=B(x, y)=-(x-y)^{2}, \hat{\eta}(x, z)=x-z, \bar{\eta}(y, w)=y-w, f(x, y)=x^{2}-y^{2}$, and $g(x, y)=y^{2}-x^{2}$ for all $x, z \in C$ and $y, w \in D$. Obviously, the conditions (i)-(v) of Theorem 3.4 are satisfied. Note that

$$
\begin{aligned}
& \{(x, y) \in C \times D: d(x, S(x, y)) \leq \varepsilon,\langle A(x, y), \widehat{\eta}(x, z)\rangle+f(x, y)-f(z, y) \\
& \left.\quad \leq \varepsilon+\frac{\alpha}{2}\|x-z\|^{2}, \forall z \in S(x, y)\right\}
\end{aligned}
$$


Journal of Applied Mathematics

$$
\begin{aligned}
= & \left\{(x, y) \in C \times D: d(x, S(x, y)) \leq \varepsilon,-(x-y)^{2}(x-z)+x^{2}-z^{2}\right. \\
& \left.\leq \varepsilon+\frac{\alpha}{2}(x-z)^{2}, \forall z \in S(x, y)\right\} \\
= & \left\{(x, y) \in C \times D: d(x, S(x, y)) \leq \varepsilon,-(x-y)^{2}(x-z)\right. \\
& \left.-(2+\alpha)\left(z-\frac{\alpha x}{2+\alpha}\right)^{2}+\frac{4}{2+\alpha} x^{2}-2 \varepsilon \leq 0, \forall z \in S(x, y)\right\} \\
= & {\left[0, \sqrt{\frac{(2+\alpha) \varepsilon}{2}}\right] \times \mathbf{R}, } \\
\{(x, y) \in & C \times D: d(y, T(x, y)) \leq \varepsilon,\langle B(x, y), \bar{\eta}(y, w)\rangle+g(x, y)-g(x, w) \\
\leq \varepsilon+ & \left.\frac{\alpha}{2}\|y-w\|^{2}, \forall w \in T(x, y)\right\} \\
= & \left\{(x, y) \in C \times D: d(y, T(x, y)) \leq \varepsilon,-(x-y)^{2}(y-w)+y^{2}-w^{2}\right. \\
& \left.\leq \varepsilon+\frac{\alpha}{2}(y-w)^{2}, \forall w \in T(x, y)\right\} \\
= & \left\{(x, y) \in C \times D: d(y, T(x, y)) \leq \varepsilon,-(x-y)^{2}(y-w)\right. \\
& \left.-(2+\alpha)\left(w-\frac{\alpha y}{2+\alpha}\right)^{2}+\frac{4}{2+\alpha} y^{2}-2 \varepsilon \leq 0, \forall w \in T(x, y)\right\} \\
& \mathbf{R} \times\left[0, \sqrt{\frac{(2+\alpha) \varepsilon}{2}}\right] .
\end{aligned}
$$

It follows that

$$
\Omega_{\alpha}(\varepsilon)=\left[0, \sqrt{\frac{(2+\alpha) \varepsilon}{2}}\right] \times\left[0, \sqrt{\frac{(2+\alpha) \varepsilon}{2}}\right]
$$

and so diam $\Omega_{\alpha} \rightarrow 0$ as $\varepsilon \rightarrow 0$. By Theorem 3.4, SMQVLI (2.1) is $\alpha$-well-posed.

Example 3.7. Let $X=Y=\mathbf{R}$ and $C=D=\mathbf{R}^{+}(=[0,+\infty))$. Let $S(x, y)=[0, x], T(x, y)=[0, y]$, $A(x, y)=B(x, y)=-(x-y)^{2}, \hat{\eta}(x, z)=x-z, \bar{\eta}(y, w)=y-w$, and $f(x, y)=g(x, y)=-x y$ for all $x, z \in C$ and $y, w \in D$. It is easy to see that the conditions (i)-(v) of Theorem 3.4 are satisfied, and $\Omega_{\alpha}(\varepsilon)=[0,+\infty) \times[0,+\infty)$. But, SMQVLI (2.1) is not $\alpha$-well-posed, since $\operatorname{diam} \Omega_{\alpha}(\varepsilon) \nrightarrow 0$ as $\varepsilon \rightarrow 0$.

Whenever $\alpha=0$, we have the following result. 
Theorem 3.8. Assume that the following conditions hold:

(i) set-valued mappings $S$ and $T$ are nonempty convex-valued, $(s, w)$-closed, $(s, s)$-lower semicontinuous, and $(s, w)$-subcontinuous on $C \times D$;

(ii) single-valued mappings $A$ and $B$ are $\left(s, w^{*}\right)$-continuous on $C \times D$;

(iii) single-valued mappings $\hat{\eta}$ and $\bar{\eta}$ are Lipschitz continuous with constants $\hat{\lambda}$ and $\bar{\lambda}$, respectively, such that for all $x_{1}, x_{2}, x_{3} \in C$ and $y_{1}, y_{2}, y_{3} \in D$ :

$$
\widehat{\eta}\left(x_{1}, x_{3}\right)=\widehat{\eta}\left(x_{1}, x_{2}\right)+\widehat{\eta}\left(x_{2}, x_{3}\right), \quad \bar{\eta}\left(y_{1}, y_{3}\right)=\bar{\eta}\left(y_{1}, y_{2}\right)+\bar{\eta}\left(y_{2}, y_{3}\right) ;
$$

(iv) functions $f$ and $g$ are continuous on $C \times D$.

Then, SMQVLI (2.1) is well-posed if and only if

$$
\Omega_{0}(\varepsilon) \neq \emptyset, \quad \forall \varepsilon>0, \quad \lim _{\varepsilon \rightarrow 0} \operatorname{diam} \Omega_{0}(\varepsilon)=0 .
$$

Corollary 3.9 (i.e., [35, Corollary 3.1]). Assume that the following conditions hold:

(i) set-valued mappings $S$ and $T$ are nonempty convex-valued, $(s, w)$-closed, $(s, s)$-lower semicontinuous, and $(s, w)$-subcontinuous on $C \times D$;

(ii) functions $f$ and $g$ are continuous on $C \times D$.

Then, SQEP (2.2) is well-posed if and only if

$$
M_{\varepsilon} \neq \emptyset, \quad \forall \varepsilon>0, \quad \lim _{\varepsilon \rightarrow 0} \operatorname{diam} M_{\varepsilon}=0 .
$$

The following theorem shows that under some suitable conditions, the $\alpha$-wellposedness of SMQVLI (2.1) is equivalent to the existence and uniqueness of its solutions.

Theorem 3.10. Let $X$ and $Y$ be two finite-dimensional spaces. Suppose that the following conditions hold:

(i) set-valued mappings $S$ and $T$ are nonempty convex-valued, closed, lower semicontinuous, and subcontinuous on $C \times D$;

(ii) single-valued mappings $A$ and $B$ are continuous on $C \times D$;

(iii) single-valued mappings $\hat{\eta}$ and $\bar{\eta}$ are Lipschitz continuous with constants $\hat{\lambda}$ and $\bar{\lambda}$ respectively, such that

(a) $\hat{\eta}\left(x_{1}, x_{3}\right)=\widehat{\eta}\left(x_{1}, x_{2}\right)+\widehat{\eta}\left(x_{2}, x_{3}\right)$ for all $x_{1}, x_{2}, x_{3} \in C$ and $\bar{\eta}\left(y_{1}, y_{3}\right)=\bar{\eta}\left(y_{1}, y_{2}\right)+$ $\bar{\eta}\left(y_{2}, y_{3}\right)$ for all $y_{1}, y_{2}, y_{3} \in D$,

(b) $\hat{\eta}(\cdot, \cdot)$ and $\bar{\eta}(\cdot, \cdot)$ both are affine in the second variable;

(iv) the functions $f$ and $g$ are continuous on $C \times D$;

(v) for any $y \in D$, the function $f(\cdot, y)$ is convex on $C$; for any $x \in C$, the function $g(x, \cdot)$ is convex on $D$;

(vi) $\Omega_{\alpha}(\varepsilon)$ is nonempty bounded for some $\varepsilon>0$.

Then, SMQVLI (2.1) is $\alpha$-well-posed if and only if SMQVLI (2.1) has a unique solution. 
Proof. The necessary of the theorem is obvious. In order to show the sufficiency, let $\left(x_{0}, y_{0}\right)$ be the unique solution of SMQVLI (2.1) and let $\left\{\left(x_{n}, y_{n}\right)\right\}$ be any $\alpha$-approximating sequence for SMQVLI (2.1). Then there exists a sequence $\varepsilon_{n}>0$ with $\varepsilon_{n} \rightarrow 0$ such that

$$
\begin{array}{r}
d\left(x_{n}, S\left(x_{n}, y_{n}\right)\right) \leq \varepsilon_{n}, \quad\left\langle A\left(x_{n}, y_{n}\right), \widehat{\eta}\left(x_{n}, z\right)\right\rangle+f\left(x_{n}, y_{n}\right)-f\left(z, y_{n}\right) \leq \varepsilon_{n}+\frac{\alpha}{2}\left\|x_{n}-z\right\|^{2}, \\
\forall z \in S\left(x_{n}, y_{n}\right), \\
d\left(y_{n}, T\left(x_{n}, y_{n}\right)\right) \leq \varepsilon_{n}, \quad\left\langle B\left(x_{n}, y_{n}\right), \bar{\eta}\left(y_{n}, w\right)\right\rangle+g\left(x_{n}, y_{n}\right)-g\left(x_{n}, w\right) \leq \varepsilon_{n}+\frac{\alpha}{2}\left\|y_{n}-w\right\|^{2}, \\
\forall w \in T\left(x_{n}, y_{n}\right),
\end{array}
$$

which means $\left\{\left(x_{n}, y_{n}\right)\right\} \subset \Omega_{\alpha}\left(\varepsilon_{n}\right)$, for all $n \in N$. Let $\varepsilon>0$ be such that $\Omega_{\alpha}(\varepsilon)$ is nonempty bounded. Then there exists $n_{0} \in N$ such that $\left\{\left(x_{n}, y_{n}\right)\right\} \subset \Omega_{\alpha}\left(\varepsilon_{n}\right) \subset \Omega_{\alpha}(\varepsilon)$ for all $n \geq n_{0}$. Thus, $\left\{\left(x_{n}, y_{n}\right)\right\}$ is bounded and so the sequence $\left\{\left(x_{n}, y_{n}\right)\right\}$ has a subsequence $\left\{\left(x_{n_{k}}, y_{n_{k}}\right)\right\}$ which converges to $(\tilde{x}, \tilde{y})$. Reasoning as in Theorem 3.4 , one can prove that $(\tilde{x}, \tilde{y})$ solves SMQVLI (2.1). The uniqueness of the solution implies that $\left(x_{0}, y_{0}\right)=(\tilde{x}, \tilde{y})$, and so the whole sequence $\left\{\left(x_{n}, y_{n}\right)\right\}$ converges to $\left(x_{0}, y_{0}\right)$. Thus, SMQVLI (2.1) is $\alpha$-well-posed. This completes the proof.

Example 3.11. Let $X=Y=\mathbf{R}$ and $C=D=\mathbf{R}^{+}(=[0,+\infty))$. Let $S(x, y)=[0, x], T(x, y)=[0, y]$, $A(x, y)=B(x, y)=-(x-y)^{2}, \widehat{\eta}(x, z)=x-z, \bar{\eta}(y, w)=y-w, f(x, y)=x^{2}-y^{2}$, and $g(x, y)=$ $y^{2}-x^{2}$ for all $x, z \in C$ and $y, w \in D$. Clearly, the conditions (i)-(vi) of Theorem 3.8 are satisfied, and SMQVLI (2.1) has a unique solution $\left(x_{0}, y_{0}\right)=(0,0)$. By Theorem 3.8, SMQVLI (2.1) is $\alpha$-well-posed.

Corollary 3.12 (i.e., [35, Theorem 3.3]). Let $X$ and $Y$ be two finite-dimensional spaces. Suppose that the following conditions hold:

(i) set-valued mappings $S$ and $T$ are nonempty convex-valued, closed, lower semicontinuous and subcontinuous on $C \times D$;

(ii) the functions $f$ and $g$ are continuous on $C \times D$;

(iii) for any $y \in D$, the function $f(\cdot, y)$ is convex on $C$; for any $x \in C$, the function $g(x, \cdot)$ is convex on $D$;

(iv) $M_{\varepsilon}$ is nonempty bounded for some $\varepsilon>0$.

Then, SQEP (2.2) is $\alpha$-well-posed if and only if SQEP (2.2) has a unique solution.

\section{Metric Characterizations of $\alpha$-Well-Posedness in the Generalized Sense for SMQVLI}

In this section, we derive some metric characterizations of $\alpha$-well-posedness in the generalized sense for SMQVLI (2.1) by considering the noncompactness of approximate solution set. 
Theorem 4.1. SMQVLI (2.1) is $\alpha$-well-posed in the generalized sense if and only if the solution set $\Gamma$ of SMQVLI (2.1) is nonempty compact and

$$
e\left(\Omega_{\alpha}(\varepsilon), \Gamma\right) \longrightarrow 0 \quad \text { as } \varepsilon \longrightarrow 0 .
$$

Proof. Suppose that SMQVLI (2.1) is $\alpha$-well-posed in the generalized sense. Then $\Gamma$ is nonempty. To show the compactness of $\Gamma$, let $\left\{\left(x_{n}, y_{n}\right)\right\} \subset \Gamma$. Clearly, if $\left\{\left(x_{n}, y_{n}\right)\right\}$ is an approximation sequence of SMQVLI (2.1), then it is also $\alpha$-approximation sequence. Since SMQVLI (2.1) is $\alpha$-well-posed in the generalized sense, it contains a subsequence converging strongly to an element of $\Gamma$. Thus, $\Gamma$ is compact. Now, we prove that (4.1) holds. Suppose by contradiction that there exist $\gamma>0,0<\varepsilon_{n} \rightarrow 0$, and $\left(x_{n}, y_{n}\right) \in \Omega_{\alpha}\left(\varepsilon_{n}\right)$ such that

$$
d\left(\left(x_{n}, y_{n}\right), \Gamma\right) \geq \gamma
$$

Being $\left\{\left(x_{n}, y_{n}\right)\right\} \subset \Omega_{\alpha}\left(\varepsilon_{n}\right),\left\{\left(x_{n}, y_{n}\right)\right\}$ is an $\alpha$-approximating sequence for SMQVLI (2.1). Since SMQVLI (2.1) is $\alpha$-well-posed in the generalized sense, there exists a subsequence $\left\{\left(x_{n_{k}}, y_{n_{k}}\right)\right\}$ of $\left\{\left(x_{n}, y_{n}\right)\right\}$ converging strongly to some element of $\Gamma$. This contradicts (4.2) and so (4.1) holds.

To prove the converse, suppose that $\Gamma$ is nonempty compact and (4.1) holds. Let $\left\{\left(x_{n}, y_{n}\right)\right\}$ be an $\alpha$-approximating sequence for SMQVLI (2.1). Then $\left\{\left(x_{n}, y_{n}\right)\right\} \subset \Omega_{\alpha}\left(\varepsilon_{n}\right)$, and so $e\left(\Omega_{\alpha}\left(\varepsilon_{n}\right), \Gamma\right) \rightarrow 0$. This implies that there exists a sequence $\left\{\left(z_{n}, w_{n}\right)\right\} \subset \Gamma$ such that

$$
\left\|\left(x_{n}, y_{n}\right)-\left(z_{n}, w_{n}\right)\right\| \longrightarrow 0,
$$

where the norm $\|\cdot\|$ in the product space $X \times Y$ is defined as follows:

$$
\|(u, v)-(\bar{u}, \bar{v})\|=\sqrt{\|u-\bar{u}\|^{2}+\|v-\bar{v}\|^{2}}, \quad \forall(u, v),(\bar{u}, \bar{v}) \in X \times Y .
$$

(It is not hard to verify that $X \times Y$ is a Banach space in terms of the last norm.)

Since $\Gamma$ is compact, there exists a subsequence $\left\{\left(z_{n_{k}}, w_{n_{k}}\right)\right\}$ of $\left\{\left(z_{n}, w_{n}\right)\right\}$ converging strongly to $\left(x_{0}, y_{0}\right) \in \Gamma$. Hence the corresponding subsequence $\left\{\left(x_{n_{k}}, y_{n_{k}}\right)\right\}$ of $\left\{\left(x_{n}, y_{n}\right)\right\}$ converges strongly to $\left(x_{0}, y_{0}\right)$. Therefore, SMQVLI (2.1) is $\alpha$-well-posed in the generalized sense.

We give the following example to illustrate that the compactness condition of $\Gamma$ is necessary.

Example 4.2. Let $X=Y=\mathbf{R}$ and $C=D=\mathbf{R}^{+}(=[0,+\infty))$. Let $S(x, y)=[x, x+y], T(x, y)=$ $[y, x+y], A(x, y)=B(x, y)=x^{2}+y^{2}, \widehat{\eta}(x, z)=x-z, \bar{\eta}(y, w)=y-w$, and $f(x, y)=$ $g(x, y)=x y$ for all $x, z \in C$ and $y, w \in D$. Then $\Gamma=\Omega_{\alpha}(\varepsilon)=[0,+\infty) \times[0,+\infty)$. It is clear that $e\left(\Omega_{\alpha}(\varepsilon), \Gamma\right) \rightarrow 0$ as $\varepsilon \rightarrow 0$. It is easy to see that the diverging sequence $\{(n, n)\}_{n \in N}$ is an $\alpha$-approximating sequence, but it has no convergent subsequence. Therefore, SMQVLI (2.1) is not $\alpha$-well-posed in the generalized sense. 
Corollary 4.3 (i.e., [35, Theorem 4.1]). SQEP (2.2) is $\alpha$-well-posed in the generalized sense if and only if the solution set $\Gamma$ of $S Q E P(2.2)$ is nonempty compact and

$$
e\left(M_{\varepsilon}, \Gamma\right) \longrightarrow 0 \quad \text { as } \varepsilon \longrightarrow 0
$$

Theorem 4.4. Assume that the following conditions hold:

(i) set-valued mappings $S$ and $T$ are nonempty convex-valued, $(s, w)$-closed, $(s, s)$-lower semicontinuous, and $(s, w)$-subcontinuous on $C \times D$;

(ii) single-valued mappings $A$ and $B$ are $\left(s, w^{*}\right)$-continuous on $C \times D$;

(iii) single-valued mappings $\hat{\eta}$ and $\bar{\eta}$ are Lipschitz continuous with constants $\hat{\lambda}$ and $\bar{\lambda}$ respectively, such that

(a) $\hat{\eta}\left(x_{1}, x_{3}\right)=\hat{\eta}\left(x_{1}, x_{2}\right)+\hat{\eta}\left(x_{2}, x_{3}\right)$ for all $x_{1}, x_{2}, x_{3} \in C$ and $\bar{\eta}\left(y_{1}, y_{3}\right)=\bar{\eta}\left(y_{1}, y_{2}\right)+$ $\bar{\eta}\left(y_{2}, y_{3}\right)$ for all $y_{1}, y_{2}, y_{3} \in D$,

(b) $\widehat{\eta}(\cdot, \cdot)$ and $\bar{\eta}(\cdot, \cdot)$ both are affine in the second variable;

(iv) functions $f$ and $g$ are continuous on $C \times D$;

(v) for any $y \in D$, the function $f(\cdot, y)$ is convex on $C$; for any $x \in C$, the function $g(x, \cdot)$ is convex on $D$.

Then, SMQVLI (2.1) is $\alpha$-well-posed in the generalized sense if and only if

$$
\Omega_{\alpha}(\varepsilon) \neq \emptyset, \quad \forall \varepsilon>0, \quad \lim _{\varepsilon \rightarrow 0} \mu\left(\Omega_{\alpha}(\varepsilon)\right)=0 .
$$

Proof. Suppose that SMQVLI (2.1) is $\alpha$-well-posed in the generalized sense. By the same argument as in Theorem 4.1, $\Gamma$ is nonempty compact, and $e\left(\Omega_{\alpha}(\varepsilon), \Gamma\right) \rightarrow 0$ as $\varepsilon \rightarrow 0$. Clearly $\Omega_{\alpha}(\varepsilon) \neq \emptyset$ for any $\varepsilon>0$, because $\Gamma \subset \Omega_{\alpha}(\varepsilon)$. Observe that for any $\varepsilon>0$, we have

$$
\mathscr{H}\left(\Omega_{\alpha}(\varepsilon), \Gamma\right)=\max \left\{e\left(\Omega_{\alpha}(\varepsilon), \Gamma\right), e\left(\Gamma, \Omega_{\alpha}(\varepsilon)\right)\right\}=e\left(\Omega_{\alpha}(\varepsilon), \Gamma\right) .
$$

Since $\Gamma$ is compact, $\mu(\Gamma)=0$ and the following relation holds (see, e.g, [2]):

$$
\mu\left(\Omega_{\alpha}(\varepsilon)\right) \leq 2 \mathcal{\ell}\left(\Omega_{\alpha}(\varepsilon), \Gamma\right)+\mu(\Gamma)=2 \not{\ell}\left(\Omega_{\alpha}(\varepsilon), \Gamma\right)=2 e\left(\Omega_{\alpha}(\varepsilon), \Gamma\right) .
$$

It follows that (4.6) holds.

Conversely, suppose that (4.6) holds. It is easy to prove that $\Omega_{\alpha}(\varepsilon)$, for any $\varepsilon>0$, is closed. Note that $\Omega_{\alpha}(\varepsilon) \subset \Omega_{\alpha}\left(\varepsilon^{\prime}\right)$ whenever $\varepsilon<\varepsilon^{\prime}$, their intersection $\Omega_{\alpha}=\bigcap_{\varepsilon>0} \Omega_{\alpha}(\varepsilon)$ is nonempty compact and satisfies $\lim _{\varepsilon \rightarrow 0} \mathscr{\ell}\left(\Omega_{\alpha}(\varepsilon), \Omega_{\alpha}\right)=0$ [39, page 412], where

$$
\begin{aligned}
\Omega_{\alpha}=\{ & \left(x_{0}, y_{0}\right) \in C \times D: x_{0} \in S\left(x_{0}, y_{0}\right),\left\langle A\left(x_{0}, y_{0}\right), \widehat{\eta}\left(x_{0}, z\right)\right\rangle+f\left(x_{0}, y_{0}\right)-f\left(z, y_{0}\right) \\
\leq & \frac{\alpha}{2}\left\|x_{0}-z\right\|^{2}, \forall z \in S\left(x_{0}, y_{0}\right), y_{0} \in T\left(x_{0}, y_{0}\right),\left\langle B\left(x_{0}, y_{0}\right), \bar{\eta}\left(y_{0}, w\right)\right\rangle \\
& +g\left(x_{0}, y_{0}\right)-g\left(x_{0}, w\right) \\
\leq & \left.\frac{\alpha}{2}\left\|y_{0}-w\right\|^{2}, \forall w \in T\left(x_{0}, y_{0}\right)\right\} .
\end{aligned}
$$


By Lemma 2.9, we obtain that $\Omega_{\alpha}$ coincides with the solution set $\Gamma$ of SMQVLI (2.1). Thus, $\Gamma$ is compact.

Let $\left\{\left(x_{n}, y_{n}\right)\right\}$ be any $\alpha$-approximating sequence for SMQVLI (2.1). Then there exists a sequence $\varepsilon_{n}>0$ with $\varepsilon_{n} \rightarrow 0$ such that

$$
\begin{array}{r}
d\left(x_{n}, S\left(x_{n}, y_{n}\right)\right) \leq \varepsilon_{n}, \quad\left\langle A\left(x_{n}, y_{n}\right), \widehat{\eta}\left(x_{n}, z\right)\right\rangle+f\left(x_{n}, y_{n}\right)-f\left(z, y_{n}\right) \leq \varepsilon_{n}+\frac{\alpha}{2}\left\|x_{n}-z\right\|^{2}, \\
\forall z \in S\left(x_{n}, y_{n}\right), \\
d\left(y_{n}, T\left(x_{n}, y_{n}\right)\right) \leq \varepsilon_{n}, \quad\left\langle B\left(x_{n}, y_{n}\right), \bar{\eta}\left(y_{n}, w\right)\right\rangle+g\left(x_{n}, y_{n}\right)-g\left(x_{n}, w\right) \leq \varepsilon_{n}+\frac{\alpha}{2}\left\|y_{n}-w\right\|^{2}, \\
\forall w \in T\left(x_{n}, y_{n}\right),
\end{array}
$$

which means $\left\{\left(x_{n}, y_{n}\right)\right\} \subset \Omega_{\alpha}\left(\varepsilon_{n}\right), \forall n \in N$. It follows from (4.6) that there exists a sequence $\left\{\left(z_{n}, w_{n}\right)\right\} \subset \Gamma$ such that

$$
\left\|\left(x_{n}, y_{n}\right)-\left(z_{n}, w_{n}\right)\right\|=d\left(\left(x_{n}, y_{n}\right), \Gamma\right) \leq e\left(\Omega_{\alpha}\left(\varepsilon_{n}\right), \Gamma\right)=\mathscr{\ell}\left(\Omega_{\alpha}\left(\varepsilon_{n}\right), \Gamma\right) \longrightarrow 0
$$

Since $\Gamma$ is compact, there exists a subsequence $\left\{\left(z_{n_{j}}, w_{n_{j}}\right)\right\}$ of $\left\{\left(z_{n}, w_{n}\right)\right\}$ converging strongly to $\left(x_{0}, y_{0}\right) \in \Gamma$. Hence, the corresponding subsequence $\left\{\left(x_{n_{j}}, y_{n_{j}}\right)\right\}$ of $\left\{\left(x_{n}, y_{n}\right)\right\}$ converges strongly to $\left(x_{0}, y_{0}\right)$. Thus, SMQVLI (2.1) is $\alpha$-well-posed in the generalized sense.

Example 4.5. Let $X=Y=\mathbf{R}$ and $C=D=[0,1]$. Let $S(x, y)=[0, x], T(x, y)=[0, y], A(x, y)=$ $B(x, y)=-(x-y)^{2}, \widehat{\eta}(x, z)=x-z, \bar{\eta}(y, w)=y-w$, and $f(x, y)=g(x, y)=-x y$ for all $x, z \in C$ and $y, w \in D$. Obviously, the conditions (i)-(v) of Theorem 4.4 are satisfied, and $\Omega_{\alpha}(\varepsilon)=[0,1] \times[0,1]$. By Theorem 4.4, SMQVLI (2.1) is $\alpha$-well-posed in the generalized sense.

Corollary 4.6 (i.e., [35, Theorem 4.2]). Assume that the following conditions hold:

(i) set-valued mappings $S$ and $T$ are nonempty convex-valued, $(s, w)$-closed, $(s, s)$-lower semicontinuous, and $(s, w)$-subcontinuous on $C \times D$;

(ii) functions $f$ and $g$ are continuous on $C \times D$;

(iii) for any $y \in D$, the function $f(\cdot, y)$ is convex on $C$; for any $x \in C$, the function $g(x, \cdot)$ is convex on $D$.

Then, SQEP (2.2) is $\alpha$-well-posed in the generalized sense if and only if

$$
M_{\varepsilon} \neq \emptyset, \quad \forall \varepsilon>0, \quad \lim _{\varepsilon \rightarrow 0} \mu\left(M_{\varepsilon}\right)=0
$$

We now give a sufficient condition for the $\alpha$-well-posedness in the generalized sense of SMQVLI (2.1) in finite-dimensional spaces.

Theorem 4.7. Let $X$ and $Y$ be two finite-dimensional spaces. Suppose that the following conditions hold:

(i) set-valued mappings $S$ and $T$ are nonempty convex-valued, closed, lower semicontinuous, and subcontinuous on $C \times D$; 
(ii) single-valued mappings $A$ and $B$ are continuous on $C \times D$;

(iii) single-valued mappings $\hat{\eta}$ and $\bar{\eta}$ are Lipschitz continuous with constants $\hat{\lambda}$ and $\bar{\lambda}$ respectively, such that

(a) (a) $\hat{\eta}\left(x_{1}, x_{3}\right)=\hat{\eta}\left(x_{1}, x_{2}\right)+\hat{\eta}\left(x_{2}, x_{3}\right)$ for all $x_{1}, x_{2}, x_{3} \in C$ and $\bar{\eta}\left(y_{1}, y_{3}\right)=$ $\bar{\eta}\left(y_{1}, y_{2}\right)+\bar{\eta}\left(y_{2}, y_{3}\right)$ for all $y_{1}, y_{2}, y_{3} \in D$,

(b) (b) $\hat{\eta}(\cdot, \cdot)$ and $\bar{\eta}(\cdot, \cdot)$ both are affine in the second variable;

(iv) functions $f$ and $g$ are continuous on $C \times D$;

(v) for any $y \in D$, the function $f(\cdot, y)$ is convex on $C$; for any $x \in C$, the function $g(x, \cdot)$ is convex on $D$;

(vi) $\Omega_{\alpha}(\varepsilon)$ is nonempty bounded for some $\varepsilon>0$.

Then, SMQVLI (2.1) is $\alpha$-well-posed in the generalized sense.

Proof. Let $\left\{\left(x_{n}, y_{n}\right)\right\}$ be any $\alpha$-approximating sequence for SMQVLI (2.1). Then there exists a sequence $\varepsilon_{n}>0$ with $\varepsilon_{n} \rightarrow 0$ such that

$$
\begin{array}{r}
d\left(x_{n}, S\left(x_{n}, y_{n}\right)\right) \leq \varepsilon_{n}, \quad\left\langle A\left(x_{n}, y_{n}\right), \hat{\eta}\left(x_{n}, z\right)\right\rangle+f\left(x_{n}, y_{n}\right)-f\left(z, y_{n}\right) \leq \varepsilon_{n}+\frac{\alpha}{2}\left\|x_{n}-z\right\|^{2}, \\
\forall z \in S\left(x_{n}, y_{n}\right), \\
d\left(y_{n}, T\left(x_{n}, y_{n}\right)\right) \leq \varepsilon_{n}, \quad\left\langle B\left(x_{n}, y_{n}\right), \bar{\eta}\left(y_{n}, w\right)\right\rangle+g\left(x_{n}, y_{n}\right)-g\left(x_{n}, w\right) \leq \varepsilon_{n}+\frac{\alpha}{2}\left\|y_{n}-w\right\|^{2}, \\
\forall w \in T\left(x_{n}, y_{n}\right) .
\end{array}
$$

As proven in Theorem 3.8, $\left\{\left(x_{n}, y_{n}\right)\right\}$ is bounded. Then there exists a subsequence $\left\{\left(x_{n_{j}}, y_{n_{j}}\right)\right\}$ of $\left\{\left(x_{n}, y_{n}\right)\right\}$ which converges to $\left(x_{0}, y_{0}\right)$. Reasoning as in Theorem 3.4, one can prove that $\left(x_{0}, y_{0}\right)$ solves SMQVLI (2.1). Therefore, SMQVLI (2.1) is $\alpha$-well-posed in the generalized sense.

The following example shows that the nonempty boundedness of $\Omega_{\alpha}(\varepsilon)$ is necessary for some $\varepsilon>0$.

Example 4.8. Let $X=Y=\mathbf{R}$ and $C=D=\mathbf{R}^{+}(=[0,+\infty))$. Let $S(x, y)=[0, x], T(x, y)=[0, y]$, $A(x, y)=B(x, y)=-(x-y)^{2}, \widehat{\eta}(x, z)=x-z, \bar{\eta}(y, w)=y-w$, and $f(x, y)=g(x, y)=-x y$ for all $x, z \in C$ and $y, w \in D$. Clearly, the conditions (i)-(v) of Theorem 4.7 are satisfied. But $\Omega_{\alpha}(\varepsilon)=[0,+\infty) \times[0,+\infty)$ is unbounded. Therefore, SMQVLI (2.1) is not $\alpha$-well-posed in the generalized sense.

Corollary 4.9 (i.e., [35, Theorem 4.3]].). Let $X$ and $Y$ be two finite-dimensional spaces. Suppose that the following conditions hold:

(i) set-valued mappings $S$ and $T$ are nonempty convex-valued, closed, lower semicontinuous, and subcontinuous on $C \times D$;

(ii) functions $f$ and $g$ are continuous on $C \times D$; 
(iii) for any $y \in D$, the function $f(\cdot, y)$ is convex on $C$; for any $x \in C$, the function $g(x, \cdot)$ is convex on $D$;

(iv) $M_{\varepsilon}$ is nonempty bounded for some $\varepsilon>0$.

Then, SQEP (2.2) is $\alpha$-well-posed in the generalized sense.

\section{Concluding Remarks}

In this paper, we generalize the concept of $\alpha$-well-posedness to the system of mixed quasivariational-like inequalities which includes as a special case symmetric quasiequilibrium problems considered by Long and Huang [35]. It is well known that symmetric quasiequilibrium problems include equilibrium problems, Nash equilibrium problems, quasivariational inequalities, variational inequalities, and fixed-point problems as special cases. It is worth emphasizing that the results presented in [35] generalize and improve some known results in the recent literature; see, for example, [12-15, 23, 33].

Further, under some suitable conditions, we obtain some metric characterizations of $\alpha$-well-posedness for the system of mixed quasivariational-like inequalities in Banach spaces. The results presented in this paper represent the supplement, improvement, generalization, and development of Long and Huang's known results [35] in the following aspects.

(i) The symmetric quasiequilibrium problem (SQEP) in [35] is extended to develop the more general problem, that is, the system of mixed quasivariational-like inequalities (SMQVLIs) in the setting of Banach spaces. Moreover, the concept of $\alpha$-well-posedness (resp., in the generalized sense) for SQEP is extended to develop the concept of $\alpha$-well-posedness (resp., in the generalized sense) for SMQVLI.

(ii) Since the system of mixed quasivariational-like inequalities (SMQVLIs) is more general and more complicated than the symmetric quasiequilibrium problem (SQEP), the assumptions in our results are very different from the corresponding ones in [35]; see, for instance, the assumptions imposed on the single-valued mappings $A, B$ and $\widehat{\eta}, \bar{\eta}$.

(iii) The new technique of arguments are applied to deriving our main results. As a matter of fact, in the process of proving our main results, our arguments depend on the properties of the mappings $\hat{\eta}, \bar{\eta}$, the uniform boundedness principle for a family of linear continuous functionals, the completion of the Banach space $(X \times Y,\|\cdot\|)$, and so forth. For instance, see the proof of Theorem 3.4.

\section{Acknowledgment}

This research was partially supported by Grant no. NSC99-2115-M-039-001 from the National Science Council of Taiwan. This research was partially supported by the National Science Foundation of China (11071169), Innovation Program of Shanghai Municipal Education Commission (09ZZ133), and Leading Academic Discipline Project of Shanghai Normal University (DZL707). 


\section{References}

[1] A. N. Tihonov, "Stability of a problem of optimization of functionals," Akademija Nauk SSSR, vol. 6, pp. 631-634, 1966.

[2] A. L. Dontchev and T. Zolezzi, Well-Posed Optimization Problems, vol. 1543, Springer, Berlin, Germany, 1993.

[3] E. Bednarczuk and J.-P. Penot, "Metrically well-set minimization problems," Applied Mathematics and Optimization, vol. 26, no. 3, pp. 273-285, 1992.

[4] G. P. Crespi, A. Guerraggio, and M. Rocca, "Well posedness in vector optimization problems and vector variational inequalities," Journal of Optimization Theory and Applications, vol. 132, no. 1, pp. 213226, 2007.

[5] M. Furi and A. Vignoli, "About well-posed minimization problems for functionals in metric spaces," Journal of Optimization Theory and Applications, vol. 5, pp. 225-229, 1970.

[6] X. X. Huang, "Pointwise well-posedness of perturbed vector optimization problems in a vectorvalued variational principle," Journal of Optimization Theory and Applications, vol. 108, no. 3, pp. 671684, 2001.

[7] X. X. Huang and X. Q. Yang, “Generalized Levitin-Polyak well-posedness in constrained optimization," SIAM Journal on Optimization, vol. 17, no. 1, pp. 243-258, 2006.

[8] X. X. Huang and X. Q. Yang, "Levitin-Polyak well-posedness of constrained vector optimization problems," Journal of Global Optimization, vol. 37, no. 2, pp. 287-304, 2007.

[9] X. X. Huang and X. Q. Yang, "Levitin-Polyak well-posedness in generalized variational inequality problems with functional constraints," Journal of Industrial and Management Optimization, vol. 3, no. 4, pp. 571-584, 2007.

[10] T. Zolezzi, "Well-posedness criteria in optimization with application to the calculus of variations," Nonlinear Analysis. Theory, Methods E Applications, vol. 25, no. 5, pp. 437-453, 1995.

[11] Y. Yao, R. Chen, and Y.-C. Liou, "A unified implicit algorithm for solving the triple-hierarchical constrained optimization problem," Mathematical and Computer Modelling. In press.

[12] L. C. Ceng and J. C. Yao, "Well-posedness of generalized mixed variational inequalities, inclusion problems and fixed-point problems," Nonlinear Analysis. Theory, Methods \& Applications, vol. 69, no. 12, pp. 4585-4603, 2008.

[13] L. C. Ceng, N. Hadjisavvas, S. Schaible, and J. C. Yao, "Well-posedness for mixed quasivariational-like inequalities," Journal of Optimization Theory and Applications, vol. 139, no. 1, pp. 109-125, 2008.

[14] Y.-P. Fang, N.-J. Huang, and J.-C. Yao, "Well-posedness of mixed variational inequalities, inclusion problems and fixed point problems," Journal of Global Optimization, vol. 41, no. 1, pp. 117-133, 2008.

[15] M. B. Lignola, "Well-posedness and L-well-posedness for quasivariational inequalities," Journal of Optimization Theory and Applications, vol. 128, no. 1, pp. 119-138, 2006.

[16] M. B. Lignola and J. Morgan, "Approximate solutions and $\alpha$-well-posedness for variational inequalities and Nash equilibria," in Decision and Control in Management Science, G. Zaccour, Ed., pp. 367378, Kluwer Academic Publisher, Dordrecht, The Netherlands, 2001.

[17] X. X. Huang, X. Q. Yang, and D. L. Zhu, "Levitin-Polyak well-posedness of variational inequality problems with functional constraints," Journal of Global Optimization, vol. 44, no. 2, pp. 159-174, 2009.

[18] I. Del Prete, M. B. Lignola, and J. Morgan, "New concepts of well-posedness for optimization problems with variational inequality constraints," Journal of Inequalities in Pure and Applied Mathematics, vol. 4, no. 1, article 5, p. 18, 2003.

[19] M. B. Lignola and J. Morgan, "Well-posedness for optimization problems with constraints defined by variational inequalities having a unique solution," Journal of Global Optimization, vol. 16, no. 1, pp. 57-67, 2000.

[20] R. Lucchetti and F. Patrone, "A characterization of Tyhonov well-posedness for minimum problems, with applications to variational inequalities," Numerical Functional Analysis and Optimization, vol. 3, no. 4, pp. 461-476, 1981.

[21] M. A. Noor and W. Oettli, "On general nonlinear complementarity problems and quasi-equilibria," Le Matematiche, vol. 49, no. 2, pp. 313-331, 1994.

[22] Y.-C. Lin, "Finite-step relaxed hybrid steepest-descent methods for variational inequalities," Journal of Inequalities and Applications, vol. 2008, Article ID 598632, 13 pages, 2008.

[23] M. B. Lignola and J. Morgan, " $\alpha$-well-posedness for Nash equilibria and for optimization problems with Nash equilibrium constraints," Journal of Global Optimization, vol. 36, no. 3, pp. 439-459, 2006.

[24] J. Morgan, "Approximations and well-posedness in multicriteria games," Annals of Operations Research, vol. 137, pp. 257-268, 2005. 
[25] J. Yu, H. Yang, and C. Yu, “Well-posed Ky Fan's point, quasi-variational inequality and Nash equilibrium problems," Nonlinear Analysis. Theory, Methods E Applications, vol. 66, no. 4, pp. 777-790, 2007.

[26] B. Lemaire, C. Ould Ahmed Salem, and J. P. Revalski, "Well-posedness by perturbations of variational problems," Journal of Optimization Theory and Applications, vol. 115, no. 2, pp. 345-368, 2002.

[27] Y. Yao and N. Shahzad, "New methods with perturbations for non-expansive mappings in Hilbert spaces," Fixed Point Theory and Applications. In press.

[28] E. Llorens-Fuster, A. Petruşel, and J.-C. Yao, "Iterated function systems and well-posedness," Chaos, Solitons and Fractals, vol. 41, no. 4, pp. 1561-1568, 2009.

[29] A. Petruşel, I. A. Rus, and J.-C. Yao, "Well-posedness in the generalized sense of the fixed point problems for multivalued operators," Taiwanese Journal of Mathematics, vol. 11, no. 3, pp. 903-914, 2007.

[30] E. Blum and W. Oettli, "From optimization and variational inequalities to equilibrium problems," The Mathematics Student, vol. 63, no. 1-4, pp. 123-145, 1994.

[31] J. Li, N.-J. Huang, and J. K. Kim, “On implicit vector equilibrium problems," Journal of Mathematical Analysis and Applications, vol. 283, no. 2, pp. 501-512, 2003.

[32] G. Mastroeni, "Gap functions for equilibrium problems," Journal of Global Optimization, vol. 27, no. 4, pp. 411-426, 2003.

[33] Y.-P. Fang, R. Hu, and N.-J. Huang, "Well-posedness for equilibrium problems and for optimization problems with equilibrium constraints," Computers $\mathcal{E}$ Mathematics with Applications, vol. 55, no. 1, pp. 89-100, 2008.

[34] X. J. Long, N.-J. Huang, and K. L. Teo, "Levitin-Polyak well-posedness for equilibrium problems with functional constraints," Journal of Inequalities and Applications, vol. 2008, Article ID 657329, 14 pages, 2008.

[35] X.-J. Long and N.-J. Huang, "Metric characterizations of $\alpha$-well-posedness for symmetric quasiequilibrium problems," Journal of Global Optimization, vol. 45, no. 3, pp. 459-471, 2009.

[36] F. Facchinei and J. S. Pang, Finite-Dimensional Variational Inequalities and Complementarity Problems, Springer, New York, NY, USA, 2003.

[37] C. Baiocchi and A. Capelo, Variational and Quasivariational Inequalities, John Wiley \& Sons, New York, NY, USA, 1984.

[38] D. Kinderlehrer and G. Stampacchia, An Introduction to Variational Inequalities and Their Applications, vol. 88 of Pure and Applied Mathematics, Academic Press, New York, NY, USA, 1980.

[39] K. Kuratowski, Topology, vol. 1-2, Academic Press, New York, NY, USA, 1968.

[40] Q. H. Ansari and J. C. Yao, "Iterative schemes for solving mixed variational-like inequalities," Journal of Optimization Theory and Applications, vol. 108, no. 3, pp. 527-541, 2001.

[41] S. K. Berberian, Lectures in Functional Analysis and Operator Theory, Springer, New York, NY, USA, 1974. 


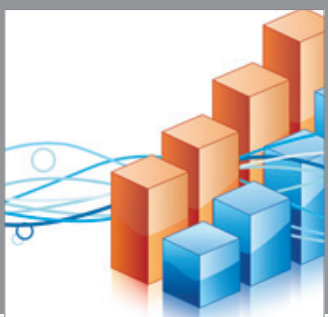

Advances in

Operations Research

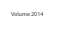

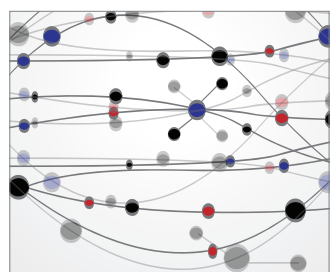

\section{The Scientific} World Journal
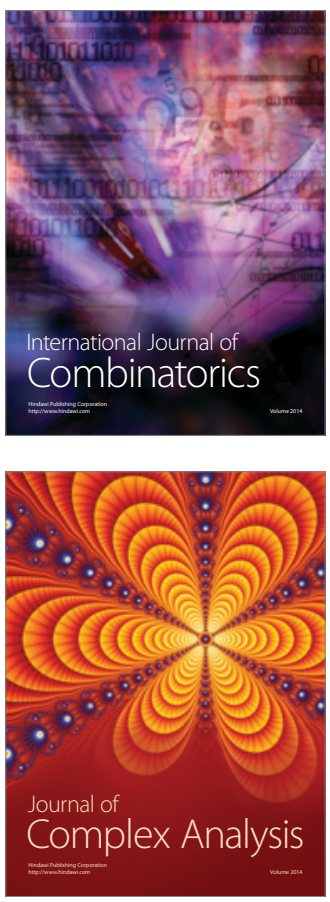

International Journal of

Mathematics and

Mathematical

Sciences
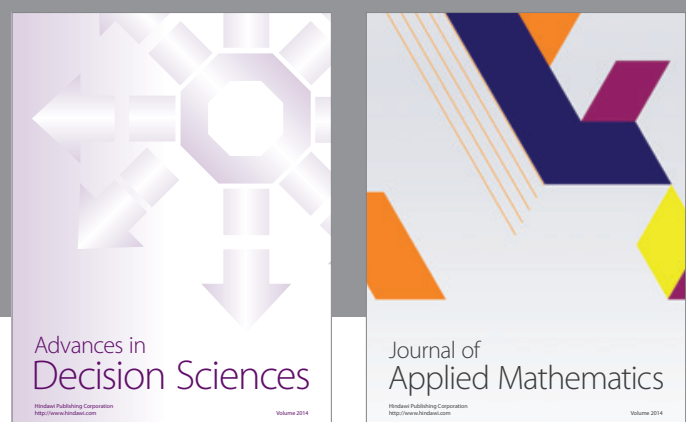

Journal of

Applied Mathematics
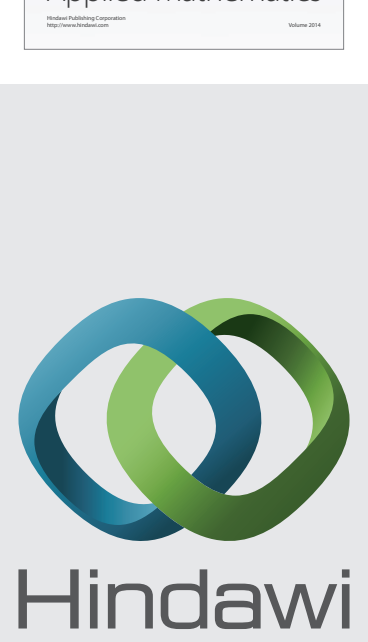

Submit your manuscripts at http://www.hindawi.com
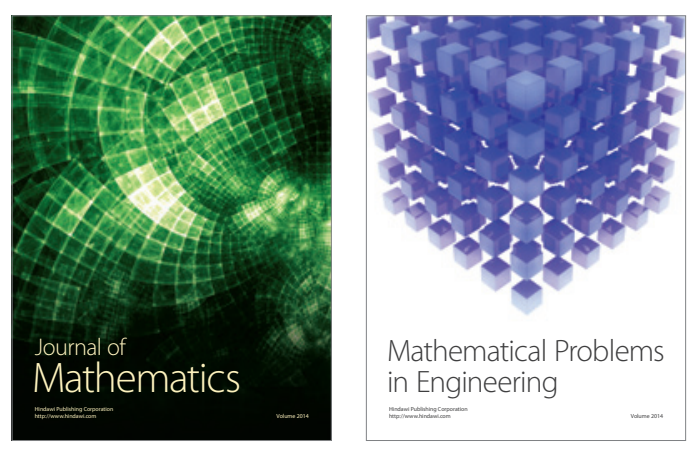

Mathematical Problems in Engineering
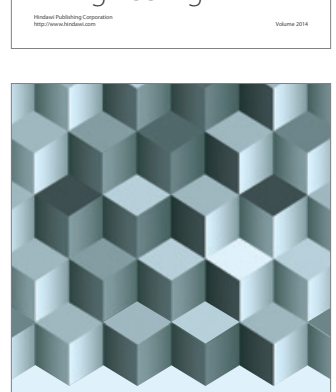

Journal of

Function Spaces
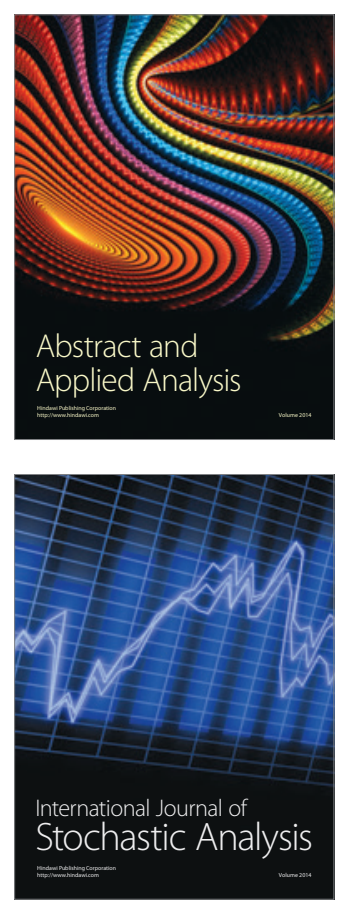

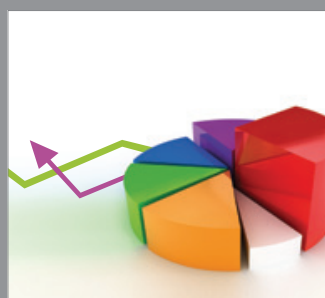

ournal of

Probability and Statistics

Promensencen
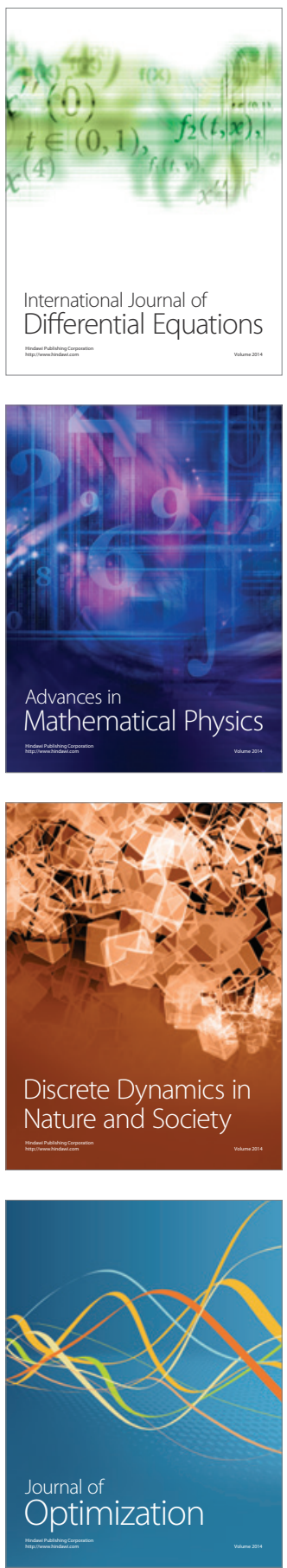